\title{
Potential Insecticidal Activity of Four Essential Oils against the Rice Weevil, Sitophilus oryzae (L.) (Coleoptera: Curculionidae)
}

\author{
Mohamed E. Tawfeek, ${ }^{\mathrm{a}}$ Hayssam M. Ali, ${ }^{\mathrm{b}}$ Mohammad Akrami, ${ }^{\mathrm{c}}$ and \\ Mohamed Z. M. Salem ${ }^{\text {d, } *}$
}

\begin{abstract}
Oils extracted from Cymbopogon citratus, Lantana camara, Artemisia camphorata, and Imperata cylindrica plants were used as potential insecticides against the rice weevil, Sitophilus oryzae (L.) (Coleoptera: Curculionidae). The phytochemical composition of the isolated oils was identified by gas chromatograph-mass spectrometry (GC-MS). Oil contact toxicities were evaluated against the adults of $S$. oryzae. The activities of acetylcholinesterase (AChE), alkaline phosphatase (ALP), and transaminases enzymes (AST) were measured. $L$. camara oil $\left(\mathrm{LC}_{50}=9.81\right.$ $\mathrm{mg} / \mathrm{cm}^{2}$ ) demonstrated the highest effect, followed by $C$. citratus oil ( $\mathrm{LC}_{50}$ $\left.=10.89 \mathrm{mg} / \mathrm{cm}^{2}\right), A$. camphorata EO $\left(\mathrm{LC}_{50}=16.12 \mathrm{mg} / \mathrm{cm}^{2}\right)$, and $I$. cylindrica oil $\left(\mathrm{LC}_{50}=36.85 \mathrm{mg} / \mathrm{cm}^{2}\right)$ against the adults of $S$. oryzae. The inhibition percentages of AChE were 38.8, 41.7, 35.0, and 27.2\%; ALP were 42.4, 49.3, 28.1, and 18.7\%; AST were 33.9, 38.7, 20.8, and 11.8\%; and ALT were 22.7, 30.5, 14.6, and $9.6 \%$ after treated $S$. oryzae with oils from C. citratus, L. camara, A. camphorata and I. cylindrica, respectively. The highest abundant compounds in $C$. citratus were geranial $(25.95 \%)$, nerylacetal $(8.85 \%)$, and neral $(8.45 \%)$, in L. camara were caryophyllene $(12.2 \%)$, and 3 -elemene $(8.89 \%)$, in $A$. camphorata were germacrene D4-ol $(20.83 \%)$, and borneol $(19.47 \%)$, and in I. cylindrica were 5phenylundecane $(10.68 \%)$, and 6-phenyldodecane $(8.70 \%)$.
\end{abstract}

Keywords: Oily extracts; Chemical composition; Contact toxicity; Sitophilus oryzae; Acetylcholinesterase; Aspartate transaminase; Alanine transaminase

Contact information: a: Department of Applied Entomology and Zoology, Faculty of Agriculture, 21545 El-Shatby, Alexandria University, Alexandria, Egypt; b: Botany and Microbiology Department, College of Science, King Saud University, P.O. Box 2455, Riyadh 11451, Saudi Arabia c: Department of Engineering, University of Exeter, Exeter EX4 4QF, UK; d: Forestry and Wood Technology Department, Faculty of Agriculture (El-Shatby), Alexandria University, Alexandria 21545, Egypt;

*Corresponding Author: zidan_forest@yahoo.com

\section{INTRODUCTION}

Stored-product pests cause critical misfortunes in weight and quality of the stored grains and cereal products (Fields and Korunic 2000; Neethirajan et al. 2007). The grain weevils (Curculionidae) are major pests of stored grains such as wheat, maize, and rice. The rice weevil, Sitophilus oryzae L. (Coleoptera: Curculionidae), is one of the most hazardous stored grain pests throughout the world (Pugazhvendan et al. 2009). Females deposit eggs into grain; larvae are legless and remain in the grain kernel for their entire duration. Feeding of S. oryzae larvae and adults can bring down grain weight by up to $75 \%$, diminishing the dietary benefit and germination of the grains resulting in lower prices for seed grain (Dal Bello et al. 2000). 
The protection of stored grain using synthetic pesticides is still the method of choice (Rattan 2010). However, many problems are associated with these chemicals, such as insect resistance, toxicity to mammals and other living organisms, toxic residues in stored products, increasing costs of application, and environmental contamination (Arthur et al. 2014). Therefore, there is interest in finding alternative ways for stored products protection. Natural pesticides with low mammalian toxicity are recommended for suppressing insect pests especially in storage (Parugrug and Roxas 2008).

Botanicals are plant-inferred materials that can be utilized as an important component in integrated pest management (IPM) for prevailing insect pests (Abdelsalam et al. 2019; Salem et al. 2020; Mosa et al. 2021; Salem et al. 2021; Moustafa et al. 2021). Botanical pesticides are biodegradable, have little or no deleterious impact on the environment and non-target living beings, cheap, easily produced, and may impede the advancement of resistance (Isman 2005; Rajendran and Sriranjini 2008). Essential oils (EOs) from different botanical parts have shown promising insecticidal activities. EOs from Cymbopogon citratus, C. nardus, and Eucalyptus citriodora have repellent effects against Anopheles arabiensis mosquitoes (Solomon et al. 2012). The EO composition of $C$. citratus EO showed the presence of neral, geranial, and $\beta$-pinene as main compounds, while in $C$. nardus they were citronellal, nerol, and citronellol; both oils showed low contact toxicity against Dinoderus porcellus L. (Coleoptera: Bostrichidae) (Loko et al. 2021). When tested against three stored grain insects, Sitophilus oryzae, Rhyzopertha dominica, and Tribolium castaneum, plant EOs could play an important role in control of stored-grain insects especially $S$. oryzae and could be recommended for use as a part of IPM program in stored grains (Tawfeek et al. 2017).

Acetylcholinesterase (AChE, EC 3.1.1.7) is a key enzyme that terminates nerve impulses by catalyzing the hydrolysis of neurotransmitter, acetylcholine, in the nervous system of various organisms. Its inhibition leads to paralysis and death (Zibaee 2011). Alkaline phosphatase (ALP, EC 3.1.3.1) is a set of hydrolytic enzymes, which hydrolyzes phosphomonoesters under alkaline conditions causing cytolysis of tissues during the insect development (Miao 2002). Aspartate aminotransferase (AST, EC 2.6.1.1) and alanine aminotransferase (ALT, EC 2.6.1.2) are also known as glutamate oxaloacetate transaminase (GOT) and glutamate pyruvate transaminase (GPT), respectively. The aminotransferases are important enzymes catalyzing amino acid catabolism and are critical in carbohydrate and protein metabolism (Zibaee et al. 2008). They are altered during various pathological and physiological activities (Etebari et al. 2005).

This work evaluated the insecticidal activities of the extracted oils from four plant species (Cymbopogon citratus, Lantana camara, Artemisia camphorata, and Imperata cylindrica) against Sitophilus oryzae (L.) adults. Their inhibitory effects on the enzyme activities of AChE, ALP, AST, and ALT were estimated.

\section{EXPERIMENTAL}

\section{Insect Rearing}

The rice weevil Sitophilus oryzae (L.) adults were gathered from infested wheat grains (Triticum aestivum L.). Insect rearing was performed at the laboratory of Applied Entomology and Zoology Department, Faculty of Agriculture (El-Shatby), Alexandria University, Alexandria, Egypt. The culture was maintained in plastic containers $(26 \times 30$ $\times 20 \mathrm{~cm})$ at $28 \pm 2{ }^{\circ} \mathrm{C}$ and $65 \pm 5 \%$ relative humidity without exposure to any insecticide. 


\section{Extraction of Plant Oils}

Fresh leaves collected from four plants and authenticated at the Department of Forestry and Wood Technology, Faculty of Agriculture, Alexandria University, Egypt, were used for the oil extraction. Oils from Cymbopogon citratus, Lantana camara, and Artemisia camphorata were extracted by the Clevenger method, where $100 \mathrm{~g}$ fresh weight from each material were cut to small pieces and put in 2-L flask containing $1000 \mathrm{~mL}$ of distilled water and subjected to hydrodistillation for $3 \mathrm{~h}$ to extract the essential oil (EO) (Salem et al. 2014, 2020). For Imperata cylindrica, $100 \mathrm{~g}$ were cut into small pieces, extracted by soaking in $\mathrm{n}$-hexane solvent $(200 \mathrm{~mL})$ for $6 \mathrm{~h}$ under shaking, and filtered with Whatman No. 1 filter paper under suction pressure (Hamada et al. 2018). The $n$-hexane oily liquid extract (HOE) was separated and concentrated by evaporating the n-hexane solvent under vacuum using a rotary evaporator at $45^{\circ} \mathrm{C}$. The HOE was stored in brown tubes (Salem et al. 2019; Mohamed et al. 2020).

\section{Contact Toxicity}

The insecticidal activity of the four oils was tested against $S$. oryzae adults by the residual film technique (Qi and Burkholder 1981), under laboratory conditions at 12:12 $\mathrm{h}$ (light: dark photoperiod). Serial dilutions of tested oils from C. citratus, L. camara, A. camphorata, and I. cylindrica at 2, 5, 10, 20,30, and $60 \mathrm{mg} / \mathrm{cm}^{2}$ were prepared in acetone as a solvent. There were four replicates for each treatment in addition to controls. One $\mathrm{mL}$ of each concentration was placed on the bottom of each Petri dish (9 cm diameter). After the acetone was evaporated, 10 adult of rice weevils were placed into each dish. The same procedure was used for the control treated with acetone. Mortality percentages were recorded after $72 \mathrm{~h}$ of treatment. The $\mathrm{LC}_{50}$ and $\mathrm{LC}_{90}$ values as well as the slope of lines were calculated according to Finney (1952). It should be noted that, according to the residual film technique used in the bioassay test, the concentration is attributed to the area Unit. A unit of area means a spread rate at which one milligram of a substance is spread over the area of one square centimeter, which used as a dose calculation unit.

\section{Biochemical Assays}

Homogenate preparation

After treatment with $\mathrm{LC}_{50}$ values of the tested oils, $S$. oryzae adults were separately weighted and homogenized in 10 volumes (w/v) of ice cold $0.1 \mathrm{M}$ phosphate buffer $(\mathrm{pH}$ 7.2) using a Teflon glass tissue homogenizer. In the ALP assay, the samples were homogenized in 10 volumes $(\mathrm{w} / \mathrm{v})$ of ice cold $0.1 \mathrm{M}$ phosphate buffer $(\mathrm{pH} 9.8)$. The homogenates were centrifuged at $5000 \mathrm{rpm}$ for $30 \mathrm{~min}$ at $4{ }^{\circ} \mathrm{C}$. The obtained supernatants were divided into small portions and stored at $-20{ }^{\circ} \mathrm{C}$. Three replicates were used for each treatment.

\section{Total soluble protein}

The total protein content was determined calorimetrically according to the method of Lowry et al. (1951) by using bovine serum albumin (BSA) as a standard.

\section{AChE activity}

Acetylcholinesterase (AChE) activity was determined according to Ellman et al. (1961). The reaction mixture contained $2.78 \mathrm{~mL}$ of $0.1 \mathrm{M}$ phosphate buffer $(\mathrm{pH} 8.0) ; 0.1$ $\mathrm{mL}$ of ten times diluted DTNB reagent solution (39.5 mg of 5, 5-dithiobis-[2-nitro-benzoic acid] and $15 \mathrm{mg}$ of sodium bicarbonate dissolved in $10 \mathrm{~mL}$ of $0.1 \mathrm{M}$ phosphate buffer, $\mathrm{pH}$ 
7.2); $0.1 \mathrm{~mL}$ of the supernatant; and $0.02 \mathrm{~mL}$ of $0.075 \mathrm{M}$ acetylthiocholine iodide. The yellow color formed was measured after $10 \mathrm{~min}$ at $412 \mathrm{~nm}$. Specific activity was expressed as $\mu \mathrm{mol}$ acetylthiocholine hydrolyzed $/ \mathrm{min} / \mathrm{mg}$ protein.

\section{Alkaline phosphatase activity}

Alkaline phosphatase (ALP) activity (Klin 1972) was determined using a diagnostic kit (Diamond Co., Cairo, Egypt). In this method, $20 \mu \mathrm{L}$ of the enzyme source was added to $1000 \mu \mathrm{L}$ of $1.0 \mathrm{M}$ diethanolamine buffer ( $\mathrm{pH} 9.8$ ) containing $0.6 \mathrm{mM}$ magnesium ions and $1 \mathrm{mM}$ p-nitrophenyl phosphate, mixed in the cuvette, incubated for $30 \mathrm{sec}$. With the stopwatch started simultaneously, the output was read again after exactly 1, 2, and 3 min at $405 \mathrm{~nm}$ using a spectrophotometer (Sequoia-Turner Model 340; Texas City, TX, USA). Specific activity was expressed as IU/mg protein/hr.

\section{Transaminases activity}

Aspartate aminotransferase (AST) and alanine aminotransferase (ALT) activities were determined as previously described (Reitman and Frankel 1957). In this method, 100 $\mu \mathrm{L}$ of enzyme source was added to $500 \mu \mathrm{L}$ of $0.1 \mathrm{M}$ phosphate buffer ( $\mathrm{pH} 7.2)$ containing $80 \mathrm{mM}$ L-aspartate as a substrate for AST or $80 \mathrm{mM}$ D-L-alanine as a substrate for ALT, and $4 \mathrm{mM}$ ó-ketoglutarate.

After incubating the mixture for $30 \mathrm{~min}$ at $37{ }^{\circ} \mathrm{C}, 500 \mu \mathrm{L}$ of developing color reagent (4 mM 2, 4-dinitrophenylhydrazine) was added, and the solution was incubated for $20 \mathrm{~min}$ at room temperature. Lastly, $5 \mathrm{~mL}$ of $0.4 \mathrm{~N} \mathrm{NaOH}$ was added, and the mixture was left at room temperature for $5 \mathrm{~min}$. An assay mixture without enzyme source was used as the blank, and the absorption was measured at $546 \mathrm{~nm}$. AST and ALT specific activities were expressed as IU/mg protein/hr.

\section{GC-MS Analysis of the Oils}

The chemical composition of the extracted oils was analyzed with a Trace GC Ultraho-ISQ mass spectrometer (Thermo Scientific, Waltham, MA, USA) with a direct capillary column TG-5MS (30 $\mathrm{m} \times 0.25 \mathrm{~mm} \times 0.25 \mu \mathrm{m}$ film thickness). Oils were diluted in $n$-hexane solvent ( $3 n$-hexane: 1 oil) before being injected to the GC-MS. The used carrier gas was He (flow rate of $1 \mathrm{~mL} / \mathrm{min}$ ).

The program and oven temperatures conditions can be found in previous work (Moustafa et al. 2021). The Xcalibur 3.0 data system (Thermo Fisher Scientific) with the Match factor from the GC-MS literature is a very intelligent tool to identify chemical constituents, where the value $\geq 650$ is acceptable to confirm the compounds (El-Sabrout $e t$ al. 2019; Behiry et al. 2020; Salem et al. 2020; Abd-Elkader et al. 2021; Ali et al. 2021; Moustafa et al. 2021).

\section{Statistical Analysis}

Means were compared for significance using one-way analysis of variance (ANOVA) test with Least Significant Difference (LSD 0.05 ) (2005). Mortality rates were corrected according to Abbott's formula (Abbott 1925) and plotted against concentrations as log/probit regression lines. LC50, LC90 values, and the toxicity index, as well as the slope of the lines were calculated according to Finney (1952). 


\section{RESULTS AND DISCUSSION}

\section{Insecticidal Activity of the Extracted Oils}

The effects of four natural plant oils from Cymbopogon citratus, Lantana camara, Artemisia camphorata, and Imperata cylindrica on the rice weevil Sitophilus oryzae adults were tested after $72 \mathrm{~h}$ from treatment by residual film technique. As shown in Table 1, among the four natural plant oils used, the L. camara EO exhibited the highest effect against the adults of $S$. oryzae $\left(\mathrm{LC}_{50}=9.81 \mathrm{mg} / \mathrm{cm}^{2}\right)$, followed by $C$. citratus $\mathrm{EO}\left(\mathrm{LC}_{50}=\right.$ $\left.10.89 \mathrm{mg} / \mathrm{cm}^{2}\right)$, A. camphorata $\mathrm{EO}\left(\mathrm{LC}_{50}=16.12 \mathrm{mg} / \mathrm{cm}^{2}\right)$, and $I$. cylindrica $\mathrm{HOE}\left(\mathrm{LC}_{50}=\right.$ $36.85 \mathrm{mg} / \mathrm{cm}^{2}$ ).

Table 1. Contact Toxicity of the Oils against $S$. oryzae Adults after $72 \mathrm{~h}$ from Treatment by Residual Film Technique

\begin{tabular}{|l|l|l|l|l|l|l|l|}
\hline $\begin{array}{l}\text { Oil source } \\
\begin{array}{l}\text { Cymbopogon } \\
\text { citratus }\end{array}\end{array}$ & $\begin{array}{l}\mathrm{LC}_{50}{ }^{\mathrm{a}} \\
\left(\mathrm{mg} / \mathrm{cm}^{2}\right)\end{array}$ & $\begin{array}{l}95 \% \mathrm{CL}^{\mathrm{b}} \\
\left(\mathrm{mg} / \mathrm{cm}^{2}\right)\end{array}$ & $\begin{array}{l}\mathrm{LC}_{90}{ }^{\mathrm{c}} \\
\left(\mathrm{mg} / \mathrm{cm}^{2}\right)\end{array}$ & $\begin{array}{l}95 \% \mathrm{CL} \\
\left(\mathrm{mg} / \mathrm{cm}^{2}\right)\end{array}$ & Slope $\pm \mathrm{SE}^{\mathrm{d}}$ & $\left(\mathrm{X}^{2}\right)^{\mathrm{e}}$ & $\mathrm{R}^{2}$ \\
\hline $\begin{array}{l}\text { Lantana } \\
\text { camara }\end{array}$ & 96.38 & 40.77 & $\begin{array}{l}27.08 \text { to } \\
61.37\end{array}$ & $2.27 \pm 0.09$ & 0.96 & 0.97 \\
\hline $\begin{array}{l}\text { Artemisia } \\
\text { camphorata }\end{array}$ & 16.12 & $\begin{array}{l}6.55 \text { to } \\
14.69\end{array}$ & 41.20 & $\begin{array}{l}27.52 \text { to } \\
61.68\end{array}$ & $2.32 \pm 0.09$ & 0.76 & 0.81 \\
\hline $\begin{array}{l}\text { Imperata } \\
\text { cylindrical }\end{array}$ & 36.85 & 108.04 & $\begin{array}{l}64.99 \\
179.63\end{array}$ & $1.76 \pm 0.11$ & 0.53 & 0.69 \\
\hline
\end{tabular}

a The concentration causing $50 \%$ mortality. ${ }^{\mathrm{b}}$ The concentration causing $90 \%$ mortality. ${ }^{\mathrm{c}}$ Confidence limits ${ }^{d}$ Slope of the concentration-mortality regression line \pm standard error. ${ }^{c}$ Chi square value.

\section{In vivo Effect of Oils on AChE and ALP Activities in S. oryzae Adults}

The changes in AChE and ALP enzymes activities in $S$. oryzae are displayed in Table 2. There was no significant difference in the AChE activity of $S$. oryzae adults after being treated with $\mathrm{LC}_{50}$ of four EOs. The inhibition percentages were 38.8, 41.7, 35.0, and $27.2 \%$ after treatment with Eos from C. citratus, L. camara, A. camphorata, and I. cylindrica, respectively, relative to the control. The inhibition percentages in ALP activity were $42.4,49.3,28.1$, and $18.7 \%$ after treatment with $C$. citratus, L. camara, A. camphorata, and I. cylindrica oils, respectively, relative to the control.

Table 2. Changes in Acetylcholinesterase (AChE) and Alkaline Phosphatase (ALP) Activities of $S$. oryzae Adults as Affected by LC 50 of Extracted Oils

\begin{tabular}{|c|c|c|c|c|c|}
\hline Oil source & $\begin{array}{c}\text { Conc. } \\
\left(\mathrm{mg} / \mathrm{cm}^{2}\right)\end{array}$ & $\begin{array}{l}\text { AChE activity } \\
(\mu \mathrm{mol} / \mathrm{min} / \mathrm{mg} \\
\text { protein }) \pm \mathrm{SD}^{1}\end{array}$ & $\begin{array}{l}\text { Chang } \\
\mathrm{e}^{2}(\%)\end{array}$ & $\begin{array}{l}\text { ALP activity (IU/ mg } \\
\text { protein/hr) } \pm \text { SD }\end{array}$ & $\begin{array}{c}\text { Change } \\
(\%)\end{array}$ \\
\hline Control & - & $32.0^{\mathrm{a}} \pm 2.6$ & - & $945.7^{a} \pm 8.4$ & - \\
\hline $\begin{array}{l}\text { Cymbopogon } \\
\text { citratus }\end{array}$ & 10.89 & $19.6^{b} \pm 1.5$ & -38.8 & $544.6^{d} \pm 7.2$ & -42.4 \\
\hline Lantana camara & 9.81 & $18.7^{b} \pm 0.5$ & -41.7 & $479.3^{\mathrm{e}} \pm 11.5$ & -49.3 \\
\hline $\begin{array}{c}\text { Artemisia } \\
\text { camphorata }\end{array}$ & 16.12 & $20.8^{b} \pm 0.5$ & -35.0 & $680.4^{c} \pm 10.8$ & -28.1 \\
\hline $\begin{array}{l}\text { Imperata } \\
\text { cylindrica }\end{array}$ & 36.85 & $23.3^{b} \pm 1.2$ & -27.2 & $768.5^{b} \pm 6.3$ & -18.7 \\
\hline \multicolumn{2}{|l|}{ LSD $_{0.05}$} & 4.66 & - & 16.54 & - \\
\hline
\end{tabular}

Means followed by the same letters in the same column are not significantly different at $P \leq 0.05$. ${ }^{1}$ SD means standard deviation. ${ }^{2}$ Change $(\%)=($ treated - control $) \div$ control $\times 100$. 


\section{In vivo Effect of Oils on AST and ALT Activities in S. oryzae Adults}

Table 3 shows the inhibitory effects in the activities of both AST and ALT in $S$. oryzae adults. The inhibition percentages of AST activity were 33.9, 38.7, 20.8, and 11.8\% after being treated $S$. oryzae adults with $C$. citratus, L. camara, A. camphorata, and $I$. cylindrica oils, respectively, compared with the control. Meanwhile, the inhibition percentages of ALT activity were 22.7, 30.5, 14.6, and 9.6\% after treating $S$. oryzae adults with the same previous extracts compared with the control.

Table 3. Changes in Aspartate Aminotransferase (AST) and Alanine Aminotransferase (ALT) Activities of $S$. oryzae Adults as Affected by $\mathrm{LC}_{50}$ of the Extracted Oils

\begin{tabular}{|c|c|c|c|c|c|}
\hline Oil Source & $\begin{array}{c}\text { Conc. } \\
\left(\mathrm{mg} / \mathrm{cm}^{2}\right)\end{array}$ & $\begin{array}{l}\text { AST activity (IU/ } / \\
\text { mg protein/hr) } \pm \\
\text { SD }^{1}\end{array}$ & $\begin{array}{c}\text { Change }^{2} \\
(\%)\end{array}$ & $\begin{array}{l}\text { ALT activity (IU/ mg } \\
\text { protein/hr) } \pm \mathrm{SD}\end{array}$ & $\begin{array}{c}\text { Change } \\
(\%)\end{array}$ \\
\hline Control & - & $542.2^{a} \pm 6.9$ & - & $753.0^{a} \pm 3.4$ & - \\
\hline $\begin{array}{l}\text { Cymbopogon } \\
\text { citratus }\end{array}$ & 10.89 & $358.3^{d} \pm 5.5$ & -33.9 & $582.3^{d} \pm 3.8$ & -22.7 \\
\hline Lantana camara & 9.81 & $332.4^{\mathrm{e}} \pm 6.2$ & -38.7 & $523.2^{\mathrm{e}} \pm 4.2$ & -30.5 \\
\hline $\begin{array}{c}\text { Artemisia } \\
\text { camphorata }\end{array}$ & 16.12 & $429.3^{c} \pm 6.5$ & -20.8 & $642.8^{c} \pm 5.6$ & -14.6 \\
\hline $\begin{array}{l}\text { Imperata } \\
\text { cylindrica }\end{array}$ & 36.85 & $478.5^{\mathrm{b}} \pm 5.8$ & -11.8 & $680.6^{\mathrm{b}} \pm 5.6$ & -9.6 \\
\hline \multicolumn{2}{|l|}{ LSD $_{0.05}$} & 11.35 & - & 8.44 & - \\
\hline
\end{tabular}

Means followed by the same letters in the same column are not significantly different at $P \leq 0.05$. ${ }^{1} \mathrm{SD}$ means standard deviation. ${ }^{2}$ Change $(\%)=($ treated - control $) \div$ control $\times 100$.

\section{Chemical Composition of the Oils}

Table 4 presents the chemical components of the EO from C. citratus, where the highest abundant compounds were geranial (25.95\%), nerylacetal (8.85\%), neral (8.45\%), linalool oxide (6.36\%), 2-furanmethanol (5.4\%), cis-linalool oxide (4.29\%), 10hydroxygeraniol $(3.92 \%)$, cis-verbenol (3.85\%), cis-linalool oxide (2.38\%), 4-methylvaleric acid $(2.25 \%)$, and sobrerol 8 -acetate $(2.19 \%)$.

Table 5 shows the chemical composition of the EO from L. camara corresponding to the highest abundant compounds caryophyllene (12.2\%), 3-elemene $(8.89 \%)$, Germacrene D (5.46\%),1,2-cyclopentanedione (3.73\%), 5-hydroxymethylfurfural (3.57\%), 2,3-dihydro-benzofuran (coumaran) (3.53\%), geranyl vinyl ether (2.98\%), 2,4dihydroxy-2,5-dimethyl-3(2H)-furan-3-one (2.97\%), adipic acid dimethyl ester (2.73\%), 3,7-dimethyl-2,3-epoxy-6-octenol (2.71\%), hydroxy-dimethyl ester butanedioic acid (2.69\%), 2-furylmethanol $(2.47 \%), 2(5 \mathrm{H})$-furanone $(2.11 \%)$, and 1-ethylpentyl acetate $(2.02 \%)$.

The chemical composition of the EO from A. camphorata is shown in Table 6, where the highest abundant compounds were germacrene D-4-ol (20.83\%), borneol (19.47\%), 1,8-cineole (7.71\%), longiverbenone (5.15\%), ascaridol (4.42\%), camphor (4.16\%), cyperotundone (2.86\%), cedran-8-ol (2.53\%), 7-epi-silphiperfol-5-ene (2.08\%), (E)-isovalencenol (2.04\%), $\beta$-dihydroagarofuran (2.03\%), (Z)-piperitol (1.90\%), and $\alpha$ bisabolol oxide A (1.76\%). 
Table 4. EO Composition of Cymbopogon citratus leaves by GC-MS

\begin{tabular}{|c|c|c|c|}
\hline $\begin{array}{l}\mathrm{RT} \\
(\min )\end{array}$ & Area \% & Compound name & $\begin{array}{l}\text { Match } \\
\text { factor }\end{array}$ \\
\hline 4.39 & 2.25 & 4-Methyl-valeric acid & 856 \\
\hline 4.94 & 5.4 & 2-Furanmethanol & 925 \\
\hline 5.33 & 0.24 & Edulan II & 687 \\
\hline 5.47 & 0.96 & 2,4-Dimethyl-5-methylthiopent-4-en-2-ol & 763 \\
\hline 5.82 & 0.75 & 5-Aminolevulinic acid & 831 \\
\hline 6.19 & 1.91 & 4-Methoxycoumarin & 954 \\
\hline 6.85 & 1.63 & 2,4-Dihydroxy-2,5-dimethyl-3(2H)-furan-3-one & 932 \\
\hline 7.27 & 0.91 & (11E)-10-Methyl-11-tridecenyl propionate & 635 \\
\hline 8.47 & 2.38 & cis-Linalool oxide & 642 \\
\hline 8.79 & 0.53 & Furaneol & 684 \\
\hline 9.22 & 0.61 & 1-Propanol & 893 \\
\hline 9.36 & 0.93 & 10-Methyl-E-11-tridecen-1-ol propionate & 601 \\
\hline 10.77 & 8.85 & Nerylacetal & 780 \\
\hline 10.98 & 0.39 & Methyl $(6 E, 9 E, 12 E)-6,9,12$-octadecatrienoate & 655 \\
\hline 12.14 & 0.29 & 2-(12-Pentadecynyloxy)tetrahydro-2H-pyran & 726 \\
\hline 12.46 & 0.76 & 4-Methyl itaconate & 767 \\
\hline 12.57 & 0.23 & 7-(2-Methoxyethyl)-2-oxepanone & 704 \\
\hline 12.80 & 1.45 & 2,3-Dihydro-benzofuran & 894 \\
\hline 13.72 & 0.34 & Geranyl vinyl ether & 760 \\
\hline 14.75 & 8.45 & Neral & 764 \\
\hline 14.96 & 1.18 & (2E,3Z)-2-Ethylidene-6-methyl-3,5-heptadienal & 877 \\
\hline 15.11 & 1.10 & 13,16-Octadecadienoic acid, methyl ester & 696 \\
\hline 16.01 & 3.92 & 10-Hydroxygeraniol & 915 \\
\hline 16.22 & 2.19 & Sobrerol-8-acetate & 830 \\
\hline 17.15 & 3.85 & cis-Verbenol & 800 \\
\hline 17.41 & 0.25 & Ascaridole & 785 \\
\hline 18.60 & 0.23 & 1-Methyl n-I-alpha-aspartyl-I-phenylalanate & 700 \\
\hline 19.00 & 1.33 & 6-Methyl-2-(2-oxiranyl)-5-hepten-2-ol & 716 \\
\hline 19.84 & 6.36 & Linalool oxide & 783 \\
\hline 19.96 & 0.81 & 2,4-Dimethylhexan-3-ol & 725 \\
\hline 20.31 & 25.95 & Geranial & 734 \\
\hline 21.02 & 4.29 & cis-Linalool oxide & 773 \\
\hline 26.03 & 0.86 & 9-Thiabicyclo[3.3.1]nonane-2,6-dione & 693 \\
\hline 29.43 & 0.29 & Limonen-6-ol, pivalate & 784 \\
\hline 29.90 & 0.47 & $n$-Hexadecanoic acid & 855 \\
\hline 34.32 & 1.20 & $\begin{array}{c}\text { 9-(3,3-Dimethyloxiran-2-yl)-2,7-dimethylnona-2,6- } \\
\text { dien-1-ol }\end{array}$ & 760 \\
\hline
\end{tabular}

Table 5. EO Composition from Lantana camara Leaves

\begin{tabular}{|c|c|c|c|}
\hline RT (min.) & Area \% & Compound & Match Factor \\
\hline 4.04 & 0.83 & 3-Methylcyclopentan-1,2-diol & 686 \\
\hline 4.16 & 0.39 & trans-2-Undecenoic acid & 693 \\
\hline 4.26 & 0.16 & $(E)$-4-Nonenal & 790 \\
\hline 4.48 & 1.39 & 7-Nonenoic-7,8-D2 acid, methyl ester & 703 \\
\hline 4.72 & 2.47 & 2-Furylmethanol & 826 \\
\hline 5.23 & 1.39 & 2,4-Dimethyl-5-methylthiopent-4-en-2-ol & 758 \\
\hline 5.42 & 0.59 & 2-Mercapto-1-hexanol & 736 \\
\hline 5.58 & 2.11 & 2(5H)-Furanone & 875 \\
\hline 5.72 & 0.77 & 3-Nonynoic acid & 634 \\
\hline
\end{tabular}




\begin{tabular}{|c|c|c|c|}
\hline 5.81 & 3.73 & 1,2-Cyclopentanedione & 879 \\
\hline 6.05 & 2.71 & 3,7-Dimethyl-2,3-epoxy-6-octenol & 801 \\
\hline 6.67 & 2.97 & 2,4-Dihydroxy-2,5-dimethyl-3(2H)-furan-3-one & 951 \\
\hline 6.76 & 0.16 & 2-(2-Methyl-2-Propenyloxy)Ethanol & 710 \\
\hline 7.41 & 1.43 & $p$-Menth-8-en-2-ol & 843 \\
\hline 7.72 & 0.38 & 4-Isopropyl-1,3-cyclohexanedione & 725 \\
\hline 8.84 & 1.55 & Furaneol & 705 \\
\hline 8.97 & 0.90 & 1,2-Diol-7-Octene & 640 \\
\hline 9.25 & 0.25 & $\beta$-Hydroxymyristic acid & 663 \\
\hline 9.35 & 0.38 & 1-Butoxy-2-ethylhexane & 731 \\
\hline 9.50 & 1.34 & 2,3-Dimethylfumaric acid & 794 \\
\hline 9.61 & 0.57 & Fumaric acid, 3-methylbut-3-enylundecyl ester & 713 \\
\hline 10.13 & 2.69 & Hydroxy-dimethyl ester butanedioic acid & 805 \\
\hline 10.50 & 0.71 & 4-Amino-1,5-pentandioic acid & 675 \\
\hline 10.86 & 12.2 & Caryophyllene & 806 \\
\hline 11.76 & 0.74 & 1,5-Di-O-benzoyl-2-deoxypentofuranose & 700 \\
\hline 11.93 & 0.65 & 3,5-Dihydroxy-2-methyl-4-pyrone & 794 \\
\hline 12.13 & 0.76 & 2-(12-Pentadecynyloxy)tetrahydro-2H-pyran & 728 \\
\hline 12.22 & 1.17 & Valeric acid, dodecyl ester & 683 \\
\hline 12.58 & 0.15 & exo-2-Hydroxycineole acetate & 722 \\
\hline 12.78 & 3.53 & 2,3-Dihydro-benzofuran (Coumaran) & 886 \\
\hline 13.13 & 3.57 & 5-Hydroxymethylfurfural & 867 \\
\hline 13.72 & 2.98 & Geranyl vinyl ether & 745 \\
\hline 14.12 & 0.72 & 6-Hydroxy-9-oxabicyclo[3.3.1]nonan-2-one & 668 \\
\hline 14.95 & 1.79 & $(2 E, 3 Z)-2$-Ethylidene-6-methyl-3,5-heptadienal & 659 \\
\hline 15.29 & 0.71 & Tridecanedial & 714 \\
\hline 15.35 & 0.43 & (E)-10-Heptadecen-8-ynoic acid, methyl ester & 698 \\
\hline 15.96 & 1.31 & 2,6-Dimethoxyphenol & 856 \\
\hline 16.47 & 2.02 & 1-Ethylpentyl acetate & 790 \\
\hline 16.71 & 0.17 & 3-(2-Aminoethyl)-1H-indol-5-ol & 684 \\
\hline 17.99 & 0.64 & Methyl 4,6-tetradecadiynoate & 750 \\
\hline 18.33 & 2.85 & 1-Methoxy-1,9(Z)-octadecadiene & 718 \\
\hline 18.85 & 1.47 & 2,7-Dioxa-tricyclo[4-4.0.0(3,8)]deca-4,9-diene & 724 \\
\hline 20.86 & 2.73 & Adipic acid dimethyl ester & 891 \\
\hline 22.06 & 0.70 & 2-Butyl-4-isopropyl-2-cyclohexen-1-one & 817 \\
\hline 22.22 & 0.34 & Oleic acid & 756 \\
\hline 22.63 & 0.42 & 12,15-Octadecadiynoic acid, methyl ester & 749 \\
\hline 22.82 & 0.89 & 3-Hydroxydodecanoic acid & 738 \\
\hline 22.92 & 0.78 & $\begin{array}{l}\text { 1-Acetyl-19,21-epoxy-15,16-dimethoxy- } \\
\text { aspidospermidin-17-ol }\end{array}$ & 761 \\
\hline 23.22 & 0.60 & cis-1,2-Cyclododecanediol & 740 \\
\hline 24.32 & 8.89 & 3-Elemene & 869 \\
\hline 25.39 & 5.46 & Germacrene D & 827 \\
\hline 25.88 & 1.74 & Thujopsenal & 776 \\
\hline 27.19 & 0.79 & $\begin{array}{c}\text { 9-(3,3-Dimethyloxiran-2-yl)-2,7-dimethylnona-2,6-dien- } \\
\text { 1-ol }\end{array}$ & 795 \\
\hline 28.66 & 0.30 & $E, E, Z-1,3,12-$ Nonadecatriene-5,14-diol & 750 \\
\hline 29.37 & 0.77 & Nerolidol-epoxyacetate & 842 \\
\hline 29.90 & 0.95 & Palmitic acid & 879 \\
\hline 33.11 & 0.21 & Methyl hexadecadienoate & 829 \\
\hline 33.21 & 0.70 & Octadec-9-enoic acid & 854 \\
\hline 33.65 & 0.22 & 2,3-Dihydroxypropyl palmitate & 784 \\
\hline
\end{tabular}


Table 6. EO Composition from Artemisia camphorata Leaves

\begin{tabular}{|c|c|c|c|}
\hline RT & Area \% & Compound & Match Factor \\
\hline 7.81 & 1.38 & $\alpha$-Terpinene & 910 \\
\hline 8.89 & 7.71 & 1,8-Cineole & 755 \\
\hline 9.93 & 1.44 & Artemisia ketone & 898 \\
\hline 11.04 & 0.38 & 4-Thujanol & 886 \\
\hline 11.65 & 0.56 & trans-para-2-Menthen-1-ol & 873 \\
\hline 14.11 & 19.47 & Borneol & 878 \\
\hline 14.73 & 1.33 & trans-Piperitol & 881 \\
\hline 14.91 & 1.90 & (Z)-Piperitol & 833 \\
\hline 15.47 & 1.35 & Silphiperfol-5-ene & 874 \\
\hline 16.13 & 2.08 & 7-epi-Silphiperfol-5-ene & 866 \\
\hline 16.44 & 0.79 & Bornyl acetate & 891 \\
\hline 17.12 & 2.04 & $(E)$-Isovalencenol & 686 \\
\hline 17.68 & 0.71 & $\alpha$-Patchoulene & 787 \\
\hline 18.95 & 0.64 & Isolongifolol, acetate & 745 \\
\hline 19.26 & 4.42 & Ascaridol & 800 \\
\hline 19.95 & 0.86 & Aromadendrene & 856 \\
\hline 20.83 & 4.16 & Camphor & 918 \\
\hline 21.83 & 0.89 & Guaia-3,9-diene & 788 \\
\hline 22.55 & 2.53 & Cedran-8-ol & 755 \\
\hline 23.57 & 2.03 & $\beta$-Dihydroagarofuran & 751 \\
\hline 24.31 & 0.99 & 24-Norursa-3,12-diene & 723 \\
\hline 25.52 & 2.86 & Cyperotundone & 711 \\
\hline 25.77 & 0.22 & 9-Methoxy-2,3-dihydrofuro[3,2-g]coumarin & 872 \\
\hline 26.29 & 20.83 & Germacrene D-4-ol & 869 \\
\hline 26.58 & 5.15 & Longiverbenone & 764 \\
\hline 26.78 & 0.30 & Isoaromadendrene epoxide & 753 \\
\hline 27.12 & 0.76 & $\gamma$-Himachalene & 740 \\
\hline 27.57 & 1.51 & 11,11-Dimethyl-4,8-dimethylenebicyclo[7.2.0]undecan-3-ol & 894 \\
\hline 28.13 & 1.38 & $\alpha$-Bisabolol & 892 \\
\hline 28.68 & 1.02 & $\alpha$-Santalol & 721 \\
\hline 28.95 & 1.03 & Bisabolone oxide $\mathrm{A}$ & 917 \\
\hline 29.54 & 0.28 & $\alpha$-Cedrene epoxide & 904 \\
\hline 29.72 & 0.18 & Liguhodgsonal & 867 \\
\hline 30.86 & 1.76 & $\alpha$-Bisabolol oxide A & 902 \\
\hline 32.62 & 1.01 & 1-Hydroxy-a-bisabolol oxide A acetate & 901 \\
\hline 32.74 & 0.26 & 2-Acetyl-4-(2,6-dimethylphenyl)-2h-1,4-thiazin-3(4H)-one & 844 \\
\hline 33.62 & 1.46 & 2-Butenoic acid, 4-(2-pentyl-1,3-dioxolan-2-yl)-, ethyl ester & 956 \\
\hline 36.65 & 0.23 & 8,9,10,11-Tetrahydrobenzo[a]anthracene & 901 \\
\hline 39.42 & 0.21 & $\begin{array}{l}\text { 5-Hydroxymethyl-1,3,3-trimethyl-2-(3-methyl-buta-1,3- } \\
\text { dienyl)-cyclopentanol }\end{array}$ & 681 \\
\hline
\end{tabular}

Table 7 presents the chemical compounds of I. cylindrica leaf HOE, where the highest abundant compounds were 5-phenylundecane (10.68\%), 6-phenyldodecane $(8.70 \%)$, 2-phenylundecane (5.84\%), 4-phenylundecane (5.15\%), 3-phenylundecane (5.08\%), 2-phenyldodecane (4.98\%), 3-phenyldodecane (4.65\%), 6-ethyl-5-hydroxy2,3,7-trimethoxynaphthoquinone (4.34\%), 5-phenyldecane (4.31\%), 6-phenyltridecane (4.09\%), 4-phenyldecane (3.84\%), 3-phenyldecane (3.76\%), 2-phenyldecane (3.47\%), 2phenyltridecane (3.24\%), 4-phenyltridecane (3.25\%), and 3-phenyltridecane (3.13\%). 
Table 7. EO Composition from of Imperata cylindrica Leaves

\begin{tabular}{|c|c|c|c|}
\hline RT & Area \% & Compound & Match Factor \\
\hline 14.28 & 0.15 & 1-Heptadecene & 772 \\
\hline 14.50 & 1.56 & Tridecane & 945 \\
\hline 16.89 & 0.44 & Cyclotetradecane & 916 \\
\hline 20.12 & 0.90 & 3,4-Dihydro-2H-1,5-(3"-t-butyl)benzodioxepine & 945 \\
\hline 20.62 & 4.31 & 5-Phenyldecane & 904 \\
\hline 20.84 & 3.84 & 4-Phenyldecane & 910 \\
\hline 21.25 & 3.76 & 3-Phenyldecane & 899 \\
\hline 22.11 & 3.47 & 2-Phenyldecane & 899 \\
\hline 22.96 & 10.68 & 5-Phenylundecane & 886 \\
\hline 23.24 & 5.15 & 4-Phenylundecane & 915 \\
\hline 23.45 & 0.32 & 4-phenyldodecane & 778 \\
\hline 23.70 & 5.08 & 3-Phenylundecane & 920 \\
\hline 24.51 & 5.84 & 2-Phenylundecane & 916 \\
\hline 24.75 & 0.32 & (1-methyl-1-propylpentyl)benzene & 842 \\
\hline 25.13 & 8.70 & 6-Phenyldodecane & 920 \\
\hline 25.94 & 4.65 & 3-Phenyldodecane & 908 \\
\hline 26.18 & 0.12 & Isomeric dodecylbenzene & 768 \\
\hline 26.46 & 0.87 & 1-Docosene & 949 \\
\hline 26.75 & 4.98 & 2-Phenyldodecane & 925 \\
\hline 27.20 & 4.09 & 6-Phenyltridecane & 878 \\
\hline 27.39 & 2.46 & 5-phenyltridecane & 904 \\
\hline 27.62 & 3.25 & 4-Phenyltridecane & 871 \\
\hline 28.06 & 3.13 & 3-Phenyltridecane & 875 \\
\hline 28.83 & 3.24 & 2-Phenyltridecane & 923 \\
\hline 29.45 & 4.34 & 6-Ethyl-5-hydroxy-2,3,7-trimethoxynaphthoquinone & 947 \\
\hline 29.55 & 0.16 & 4-phenyleicosane & 767 \\
\hline 30.11 & 1.09 & $n$-Hexadecanoic acid & 917 \\
\hline 30.78 & 0.15 & 2-phenyltetradecane & 822 \\
\hline 32.71 & 1.79 & Phytol & 926 \\
\hline 33.22 & 0.11 & 1-Heptatriacotanol & 818 \\
\hline 34.12 & 0.26 & Heptacos-1-ene & 933 \\
\hline 39.99 & 0.37 & Diisooctyl phthalate & 958 \\
\hline 42.33 & 0.16 & Heptacosane & 906 \\
\hline 43.78 & 0.11 & 2-(Tetradecyloxy)ethyl palmitate & 750 \\
\hline 44.13 & 0.16 & Dotriacontane & 824 \\
\hline
\end{tabular}

Among botanical extracts, oil extracts have received much attention as pest control agents (Campolo et al. 2018). They are valuable characterized by less persistence in air, low toxicity to human and animals, high volatility, and toxicity to insect pests of stored products (Batish et al. 2008).

In the present work, essential oil from L. camara showed the highest potential insecticidal activity against the adults of $S$. oryzae, followed by $C$. citratus, A. camphorata, and $I$. cylindrica with $\mathrm{LC}_{50}$ values of $9.81 \mathrm{mg} / \mathrm{cm}^{2}, 10.89 \mathrm{mg} / \mathrm{cm}^{2}, 16.12 \mathrm{mg} / \mathrm{cm}^{2}$, and 36.85 $\mathrm{mg} / \mathrm{cm}^{2}$, respectively.

Oil extracted from Cymbopogon species have shown potential repellent activity against storage insect pests, $S$. oryzae, Tribolium castaneum, Oryzaephilus surinamensis, and Sitophilus zeamais (Saljoqi et al. 2006; Olivero-Verbel et al. 2010; HernandezLambraño et al. 2015). By GC-MS, $\beta$-citral (43.63\%) and geranial (41.51\%), were the highest abundant compounds in the oil from $C$. citratus grown in Egypt (Mansour et al. 2020); also geranial (42.2\%) and neral (31.5\%), were the main compounds of the oil from 
Algerian C. citratus (Boukhatem et al. 2014), and from Brazil, where geranial ( $\alpha$-citral) and neral were main compounds (Silva et al. 2008). In the present study, germacrene D-4ol, borneol, 1,8-cineole, longiverbenone, ascaridol, and camphor were the highest abundant co compounds in A. camphorata EO. A. camphorata showed the major compounds germacrene D-4-ol, 1,8-cineole, ascaridole and borneol with percentages of 22, 12, 10, and $10 \%$, respectively in Brazilian plant (Seixas et al. 2018). A. camphorata EO showed the presence of davanone (20\%) as major component in Tunisia plant (Mohsen and Ali 2009).

The most interesting finding in this study is the potent contact toxicity of Lantana camara oil against the rice weevil, $S$. oryzae followed by $C$. citratus oil then ending with A. camphorata and Imperata cylindrica oil by residual film technique. These findings were in accordance with the results of Morya et al. (2010), which demonstrated the efficacy of L. camara (L.) powdered leaves against the stored grain insect pest Corcyra cephalonica (Stainton). Also, L. camara hexane extract could be used as protectant against maize weevil, S. zeamais Motsch. and bean weevil, Callosobruchus maculatus (F.) (Ogunsina et al. 2011).

Both $C$. citratus powder and methanol extract were promised as insecticides and can be used effectively in the management of $S$. oryzae in storage (Uwamose et al. 2017). The powdered leaves of Spear grass (I. cylindrica) could serve as a safer alternative to synthetic insecticides for the maize weevil (S. zeamais) infestation in stored maize grains (Ehisianya et al. 2019). Extracts from L. camara leaf were used for the stored maize protection against the infestation of $S$. zeamais (Ayalew 2020). Recently, Loko et al. (Loko et al. 2021) showed the good potential of $C$. citratus as both antifeedant and fumigant toxic agent against Dinoderus porcellus Lesne.

Furthermore, Stefanazzi et al. (2011) stated that L. camara and C. citratus oils can be used in IPM programmes for S. oryzae control. Bhatt et al. (2014) showed the toxicity and the significant reduction in larval weight of $2^{\text {nd }}$ and $6^{\text {th }}$ day's old larvae of Spodoptera litura as affected by L. camara (fruits). L. camara leaf extracts can be useful as a biorotional insecticide against Cadra cautella (Walker) eggs, larvae and adults (Gotyal et al. 2016). Furthermore, the aqueous extract of $C$. citratus Stapf leaf extract gave the highest protection on the maize grains against $S$. zeamais Motsch (Oboho et al. 2017).

Taxodium oil from trees grown in El-Beheira, Egypt, with its main compounds of $\alpha$-pinene, thujopsene, and cedrol followed by carvone and oils from trees grown in Alexandria, Egypt, with its main compounds germacrene D $(14.71 \%)$, borneol acetate $(6.81 \%)$, ledene oxide-(II) $(6.13 \%)$, and $\operatorname{trans}(\beta)$-caryophyllene $(6.13 \%)$ showed the potential $\mathrm{LC}_{50}$ against $S$. oryzae with values of $<50,28.52$, and $>50 \mu \mathrm{L} / \mathrm{L}$, respectively (Abdelsalam et al. 2019). Recently, the acetone extract from leaves of peach trees treated with Ag NPs (15 mL/L) showed LC50 of $955.24 \mathrm{ppm}$ against the rice weevils $S$. oryzae (Mosa et al. 2021). Essential oils from A. camphorata, A. absinthium, A. апnиa, A. dracunculus, and $A$. vulgaris showed potential activity against the melonworm Diaphania hyalinata (Linnaeus 1758) (Lepidoptera: Crambidae) larvae with different levels (Seixas et al. 2018).

The inhibitory effects of four tested oils on acetylcholinesterase (AChE) and alkaline phosphatase (ALP) activities in S. oryzae were examined in vivo. Several extracted oils have been described as inhibitors of AChE and ALP isolated from S. oryzae and other insect species (Kim et al. 2013; Abdelgaleil et al. 2016; Oboh et al. 2017). The inhibition of AChE activity may lead to the accumulation of acetylcholine at neuromuscular junctions, which ultimately led to insect mortality (Rajashekar et al. 2014). 
Transaminases link the metabolism of amino acids, lipids, and carbohydrates. Exposure to the extracted oils caused significant decrease in the AST and ALT activities in S. oryzae adults. A similar pattern of reduction in AST (14.29\%) and ALT (6.98\%) level was observed when cotton leafworm, S. littoralis, was exposed to spinosad (Abd ElMageed and Elgohary 2006).

From this perspective, the present findings could increase demand and experience in the use of these eco-friendly natural products, which could replace synthetic chemicals for management stored grain protection. Finally, these oils are recommended to be used as eco-friendly natural products and could replace synthetic chemicals for management stored grain protection.

\section{CONCLUSIONS}

1. Cymbopogon citratus, Lantana camara, Artemisia camphorate, and Imperata cylindrica oil extracts showed insecticidal potency against the adults of Sitophilus oryzae.

2. In terms of the enzymes inhibition percentages, L. camara essential oil gave the best results with AChE, ALP, AST, and ALT.

3. According to contact toxicity of the oils against $S$. oryzae adults after $72 \mathrm{~h}, \mathrm{~L}$. camara EO gave the highest effect followed by $C$. citratus EO.

\section{ACKNOWLEDGMENTS}

This research was funded by the Researchers Supporting Project (RSP-2021/123) King Saud University, Riyadh, Saudi Arabia.

\section{REFERENCES CITED}

Abbott, W. S. (1925). "A method of computing the effectiveness of an insecticide," Journal of Economic Entomology 18(2), 265-267. DOI: 10.1093/jee/18.2.265a

Abd El-Mageed, A., and Elgohary, L. (2006). "Impact of spinosad on some enzymatic activities of the cotton leafworm," Pakistan Journal of Biological Sciences 9(4), 713 716. DOI: $10.3923 / p j b s .2006 .713 .716$

Abdelgaleil, S. A. M., Mohamed, M. I. E., Shawir, M. S., and Abou-Taleb, H. K. (2016). "Chemical composition, insecticidal and biochemical effects of essential oils of different plant species from Northern Egypt on the rice weevil, Sitophilus oryzae L.," Journal of Pest Science 89(1), 219-229. DOI: 10.1007/s10340-015-0665-z

Abdelsalam, N. R., Salem, M. Z. M., Ali, H. M., Mackled, M. I., El-Hefny, M., Elshikh, M. S., and Hatamleh, A. A. (2019). "Morphological, biochemical, molecular, and oil toxicity properties of Taxodium trees from different locations," Industrial Crops and Products 139, 111515. DOI: 10.1016/j.indcrop.2019.111515

Abd-Elkader, D. Y., Salem, M. Z. M., Komeil, D. A., Al-Huqail, A. A., Ali, H. M., Salah, A. H., Akrami, M., and Hassan, H. S. (2021). "Post-harvest enhancing and Botrytis cinerea control of strawberry fruits using low cost and eco-friendly natural 
oils," Agronomy 11, 1246. DOI: 10.3390/agronomy11061246

Ali, H. M., Elgat, W. A. A. A., El-Hefny, M., Salem, M. Z. M., Taha, A. S., Al Farraj, D. A., Elshikh, M. S., Hatamleh, A. A., and Abdel-Salam, E. M. (2021). "New approach for using of Mentha longifolia L. and Citrus reticulata L. essential oils as woodbiofungicides: GC-MS, SEM, and MNDO quantum chemical studies," Materials 14, 1361. DOI: 10.3390/ma14061361.

Arthur, F. H., Campbell, J. F., and Toews, M. D. (2014). "Distribution, abundance, and seasonal patterns of stored product beetles in a commercial food storage facility," Journal of Stored Products Research 56, 21-32. DOI: 10.1016/j.jspr.2013.11.003

Ayalew, A. A. (2020). "Insecticidal activity of Lantana camara extract oil on controlling maize grain weevils," Toxicology Research and Application 4, 2397847320906491. DOI: $10.1177 / 2397847320906491$

Batish, D. R., Singh, H. P., Kohli, R. K., and Kaur, S. (2008). "Eucalyptus essential oil as a natural pesticide," Forest Ecology and Management 256(12), 2166-2174. DOI: 10.1016/j.foreco.2008.08.008

Behiry, S. I., Nasser, R. A., Abd El-Kareem, M. S. M., Ali, H. M., and Salem, M. Z. M. (2020). "Mass spectroscopic analysis, MNDO quantum chemical studies and antifungal activity of essential and recovered oil constituents of lemon-scented gum against three common molds," Processes 8, 275, DOI: 10.3390/pr8030275

Bhatt, P., Thodsare, N., and Srivastava, R. (2014). "Toxicity of some bioactive medicinal plant extracts to Asian army worm, Spodoptera litura," Journal of Applied and Natural Science 6(1), 139-143. DOI: 10.31018/jans.v6i1.390

Boukhatem, M. N., Ferhat, M. A., Kameli, A., Saidi, F., and Kebir, H. T. (2014). "Lemon grass (Cymbopogon citratus) essential oil as a potent anti-inflammatory and antifungal drugs," Libyan J Med 9, 25431-25431. DOI: 10.3402/ljm.v9.25431

Campolo, O., Giunti, G., Russo, A., Palmeri, V., and Zappalà, L. (2018). "Essential oils in stored product insect pest control," Journal of Food Quality 2018, 6906105. DOI: $10.1155 / 2018 / 6906105$

CoStat Statistical Software. (2005). Microcomputer Program Analysis Version 6.311, CoHort Software, Berkeley, CA, USA.

Dal Bello, G., Padin, S., López Lastra, C., and Fabrizio, M. (2000). "Laboratory evaluation of chemical-biological control of the rice weevil (Sitophilus oryzae L.) in stored grains," Journal of Stored Products Research 37(1), 77-84. DOI: 10.1016/S0022-474X(00)00009-6

Ehisianya, C., Ikpi, P., Okore, O., and Obeagu, I. (2019). "Assessment of plant powders as protectants of stored maize grains against maize weevil, Sitophilus zeamais (Motsch).[Coleoptera: Curculionidae]," Nigeria Agricultural Journal 50(1), 37-45.

Ellman, G. L., Courtney, K. D., Andres, V., and Featherstone, R. M. (1961). "A new and rapid colorimetric determination of acetylcholinesterase activity," Biochemical Pharmacology 7(2), 88-95. DOI: 10.1016/0006-2952(61)90145-9

El-Sabrout, A. M., Salem, M. Z. M., Bin-Jumah, M., Allam, A. A. (2019). "Toxicological activity of some plant essential oils against Tribolium castaneum and Culex pipiens larvae," Processes 7, 933, DOI: 10.3390/pr7120933

Etebari, K., Mirhoseini, S. Z. and Matindoost, L. (2005). "A study on interaspecific biodiversity of eight groups of silkworm (Bombyx mori) by biochemical markers," Insect Science 12(2), 87-94. DOI: 10.1111/j.1744-7917.2005.00010.x

Fields, P., and Korunic, Z. (2000). "The effect of grain moisture content and temperature on the efficacy of diatomaceous earths from different geographical locations against 
stored-product beetles," Journal of Stored Products Research 36(1), 1-13. DOI: 10.1016/S0022-474X(99)00021-1

Gotyal, B. S., Srivastava, C. and Walia, S. (2016). "Toxicity of Lantana camara leaf extracts against almond moth, Cadra cautella (Walker)," in: Proceedings of the National Academy of Sciences, India Section B: Biological Sciences 86(1), 199-204. DOI: $10.1007 / \mathrm{s} 40011-014-0438-0$

Hamada, H. M., Awad, M., El-Hefny, M. and Moustafa, M. A. M. (2018). "Insecticidal activity of garlic (Allium sativum) and ginger (Zingiber officinale) oils on the cotton leafworm, Spodoptera littoralis (Boisd.) (Lepidoptera: Noctuidae)," African Entomology 26(1) 84-94. DOI: 10.4001/003.026.0084

Hernandez-Lambraño, R., Pajaro-Castro, N., Caballero-Gallardo, K., Stashenko, E. and Olivero-Verbel, J. (2015). "Essential oils from plants of the genus Cymbopogon as natural insecticides to control stored product pests," Journal of Stored Products Research, 62, 81-83. DOI: 10.1016/j.jspr.2015.04.004

Isman, M. B. (2005). "Botanical insecticides, deterrents, and repellents in modern agriculture and an increasingly regulated world," Annual Review of Entomology, 51(1), 45-66. DOI: 10.1146/annurev.ento.51.110104.151146

Kim, S.-W., Kang, J., and Park, I.-K. (2013). "Fumigant toxicity of Apiaceae essential oils and their constituents against Sitophilus oryzae and their acetylcholinesterase inhibitory activity," Journal of Asia-Pacific Entomology 16(4), 443-448. DOI: 10.1016/j.aspen.2013.07.002

Klin, Z. (1972). "Recommendations of the German Society for Clinical Chemistry. Standardization of methods for the estimation of enzyme activities in biological fluids. Experimental basis for the optimized standard conditions," Zeitschrift für Klinische Chemie und Klinische Biochemie 10(6), 281-91.

Loko, Y. L. E., Medegan Fagla, S., Kassa, P., Ahouansou, C. A., Toffa, J., Glinma, B., Dougnon, V., Koukoui, O., Djogbenou, S. L., Tamò, M., et al. (2021). "Bioactivity of essential oils of Cymbopogon citratus (DC) Stapf and Cymbopogon nardus (L.) W. Watson from Benin against Dinoderus porcellus Lesne (Coleoptera: Bostrichidae) infesting yam chips," International Journal of Tropical Insect Science 41(1), 511524. DOI: $10.1007 / \mathrm{s} 42690-020-00235-3$

Lowry, O. H., Rosebrough, N. J., Farr, A.L. and Randall, R. J. (1951). "Protein measurement with the Folin phenol reagent," Journal of Biological Chemistry 193, 265-275.

Mansour, M. M. A., El-Hefny, M., Salem, M. Z. M., and Ali, H. M. (2020). "The biofungicide activity of some plant essential oils for the cleaner production of model linen fibers similar to those used in ancient Egyptian mummification," Processes 8(1), 79. DOI: 10.3390/pr8010079

Miao, Y. G. (2002). "Studies on the activity of the alkaline phosphatase in the midgut of infected silkworm, Bombyx mori L.," Journal of Applied Entomology 126(2-3), 138142. DOI: 10.1046/j.1439-0418.2002.00625.x

Mohamed, A. A., Behiry, S. I., Ali, H. M., El-Hefny, M., Salem, M. Z. M., and Ashmawy, N. A. (2020). "Phytochemical compounds of branches from P. halepensis oily liquid extract and $S$. terebinthifolius essential oil and their potential antifungal activity," Processes 8(3), 330. DOI: 10.3390/pr8030330

Mohsen, H., and Ali, F. (2009). "Essential oil composition of Artemisia herba-alba from southern Tunisia," Molecules 14(4), 1585-1594. DOI: 10.3390/molecules14041585

Morya, K., Pillai, S. and Patel, P. (2010). "Effect of powdered leaves of Lantana camara, 
Clerodendrum inerme and Citrus limon on the rice moth, Corcyra cephalonica," Bulletin of Insectology 63(2), 183-189.

Mosa, W. F. A., El-Shehawi, A. M., Mackled, M. I., Salem, M. Z. M., Ghareeb, R. Y., Hafez, E. E., Behiry, S. I., and Abdelsalam, N. R. (2021). "Productivity performance of peach trees, insecticidal and antibacterial bioactivities of leaf extracts as affected by nanofertilizers foliar application," Scientific Reports 11(1), 10205. DOI: 10.1038/s41598-021-89885-y

Moustafa, M. A. M., Awad, M., Amer, A., Hassan, N. N., Ibrahim, E. D. S., Ali, H. M., Akrami, M., and Salem, M. Z. M. (2021). "Insecticidal activity of lemongrass essential oil as an eco-friendly agent against the black cutworm Agrotis ipsilon (Lepidoptera: Noctuidae)," Insects 12(8), 737. DOI: 10.3390/insects12080737

Neethirajan, S., Karunakaran, C., Jayas, D. S. and White, N. D. G. (2007). "Detection techniques for stored-product insects in grain," Food Control 18(2), 157-162. DOI: 10.1016/j.foodcont.2005.09.008

Oboh, G., Ademosun, A. O., Olumuyiwa, T. A., Olasehinde, T. A., Ademiluyi, A. O., Adeyemo, A. C. (2017). "Insecticidal activity of essential oil from orange peels (Citrus sinensis) against Tribolium confusum, Callosobruchus maculatus and Sitophilus oryzae and its inhibitory effects on acetylcholinesterase and $\mathrm{Na}^{+} / \mathrm{K}^{+}-$ ATPase activities," Phytoparasitica 45(4), 501-508. DOI: 10.18311/jbc/2016/15540

Oboho, D., Eyo, J., Ekeh, F., Okweche, S. (2017). "Efficacy of Cymbopogon citratus Stapf leaf extract as seed protectant against Sitophilus zeamais Motschulsky (Coleoptera: Curculionidae) on stored maize (Zea mays L.)," Journal of Biological Control 30, 220-225, DOI: 10.18311/jbc/2016/15540.

Ogunsina, O., Oladimeji, M., and Lajide, L. (2011). "Insecticidal action of hexane extracts of three plants against bean weevil, Callosobruchus maculatus (F.) and maize weevil, Sitophilus zeamais Motsch," Journal of Ecology and the Natural Environment 3(1), 23-28. DOI: 10.5897/JENE.9000069

Olivero-Verbel, J., Nerio, L. S., and Stashenko, E. E. (2010). "Bioactivity against Tribolium castaneum Herbst (Coleoptera: Tenebrionidae) of Cymbopogon citratus and Eucalyptus citriodora essential oils grown in Colombia," Pest Management Science 66(6), 664-668. DOI: 10.1002/ps.1927

Parugrug, M. L., and Roxas, A. C. (2008). "Insecticidal action of five plants against maize weevil, Sitophilus zeamais Motsch. (Coleoptera: Curculionidae)," Current Applied Science and Technology 8(1), 24-38.

Finney, D. J. (1952). Probit Analysis: A Statistical Treatment of the Sigmoid Response Curve, Cambridge University Press, Cambridge.

Pugazhvendan, S., Elumalai, K., Ross, P. R., and Soundarajan, M. (2009). "Repellent activity of chosen plant species against Tribolium castaneum," World Journal of Zoology 4(3), 188-190.

Qi, Y.-T., and Burkholder, W. E. (1981). "Protection of stored wheat from the granary weevil by vegetable oils," Journal of Economic Entomology 74(5), 502-505. DOI: 10.1093/jee/74.5.502

Rajashekar, Y., Raghavendra, A., and Bakthavatsalam, N. (2014). "Acetylcholinesterase inhibition by biofumigant (coumaran) from leaves of Lantana camara in stored grain and household insect pests," BioMed Research International 2014, 187019. DOI: $10.1155 / 2014 / 187019$

Rajendran, S., and Sriranjini, V. (2008). "Plant products as fumigants for stored-product insect control," Journal of Stored Products Research 44(2), 126-135. DOI: 
10.1016/j.jspr.2007.08.003

Rattan, R. S. (2010). "Mechanism of action of insecticidal secondary metabolites of plant origin," Crop Protection 29(9), 913-920. DOI: 10.1016/j.cropro.2010.05.008

Reitman, S., and Frankel, S. (1957). "A colorimetric method for the determination of serum glutamic oxalacetic and glutamic pyruvic transaminases," American Journal of Clinical Pathology 28(1), 56-63. DOI: 10.1093/ajcp/28.1.56

Salem, M. Z. M., Ali, H. M., and Basalah, M. O. (2014). "Essential oils from wood, bark, and needles of Pinus roxburghii Sarg. from Alexandria, Egypt: Antibacterial and antioxidant activities," BioResources 9(4), 7454-7466. DOI: 10.15376/biores.9.4. 7454-7466

Salem, M. Z. M., Ali, M. F., Mansour, M. M. A., Ali, H. M., Abdel Moneim, E. M., Abdel-Megeed, A. (2020). "Anti-termitic activity of three plant extracts, chlorpyrifos, and a bioagent compound (Protecto) against termite Microcerotermes eugnathus Silvestri (Blattodea: Termitidae) in Egypt," Insects 2020, 11, 756. DOI: 10.3390/insects 11110756.

Salem, M. Z. M., El-Hefny, M., Ali, H. M., Abdel-Megeed, A., El-Settawy, A. A. A., Böhm, M., Mansour, M. M. A., and Salem, A. Z. M. (2021). "Plants-derived bioactives: Novel utilization as antimicrobial, antioxidant and phytoreducing agents for the biosynthesis of metallic nanoparticles," Microbial Pathogenesis 158, 105107. DOI: 10.1016/j.micpath.2021.105107

Salem, M. Z. M., Behiry, S. I., and EL-Hefny, M. (2019). "Inhibition of Fusarium culmorum, Penicillium chrysogenum and Rhizoctonia solani by n-hexane extracts of three plant species as a wood-treated oil fungicide," Journal of Applied Microbiology 126(6), 1683-1699. DOI: 10.1111/jam.14256

Saljoqi, A. U. R., Afridi, M. K., Khan, S. A., and Rehman, S. (2006). "Effects of six plant extracts on rice weevil Sitophilus oryzae L. in the stored wheat grains," Journal of Agricultural and Biological Science 1(4), 1-5.

Seixas, P. T. L., Demuner, A. J., Alvarenga, E. S., Barbosa, L. C. A., Marques, A., Farias, E. D .S., and Picanço, M. C. (2018). "Bioactivity of essential oils from Artemisia against Diaphania hyalinata and its selectivity to beneficial insects," Scientia Agricola 75, 519-525. DOI: 10.1590/1678-992X-2016-0461

Silva, C. d. B. d., Guterres, S. S., Weisheimer, V., and Schapoval, E. E. (2008). "Antifungal activity of the lemongrass oil and citral against Candida spp.," Brazilian Journal of Infectious Diseases 12(1), 63-66.

Solomon, B., Gebre-Mariam, T., and Asres, K. (2012). "Mosquito repellent actions of the essential oils of Cymbopogon citratus, Cymbopogon nardus and Eucalyptus citriodora: Evaluation and formulation studies," Journal of Essential Oil Bearing Plants 15(5), 766-773. DOI: 10.1080/0972060X.2012.10644118

Stefanazzi, N., Stadler, T., and Ferrero, A. (2011). "Composition and toxic, repellent and feeding deterrent activity of essential oils against the stored-grain pests Tribolium castaneum (Coleoptera: Tenebrionidae) and Sitophilus oryzae (Coleoptera: Curculionidae)," Pest Management Science 67(6), 639-646. DOI: 10.1002/ps.2102

Tawfeek, M., Abu-Shall, A., Gad, A., and Mohey, M. (2017). "Evaluation of six plant essential oils against three stored product insects and their effects on the Haemogram under laboratory conditions," Alexandria Journal of Agricultural Sciences 62(3), 291301. DOI: 10.21608/alexja.2017.5793

Uwamose, M. O., Nmor, J. C., Okulogbo, B. C., and Ake, J. E. (2017). “Toxicity of lemon grass Cymbopogon citratus powder and methanol extract against rice weevil 
Sitophilus oryzae (Coleoptera: Curculionidae)," Journal of Coastal Life Medicine 5(3), 99-103.

Zibaee, A., Sendi, J. J., Etebari, K., Alinia, F., and Ghadamyari, M. (2008). "The effect of diazinon on some biochemical characteristics of Chilo suppressalis Walker

(Lepidoptera: Pyralidae), rice striped stem borer," Mun. Entomol. Zool. 3(1), 255-265.

Zibaee, A. (2011). "Botanical insecticides and their effects on insect biochemistry and immunity," in: Pesticides in the Modern World - Pests Control and Pesticides Exposure and Toxicity Assessment, M. Stoytcheva (ed.), InTech, pp. 55-68.

Article submitted: August 31, 2021; Peer review completed: September 28, 2021;

Revised version received and accepted: September 29, 2021; Published: October 4, 2021. DOI: 10.15376/biores.16.4.7767-7783 